\title{
Article \\ Haze Removal Based on Refined Transmission Map for Aerial Image Matching
}

\author{
Yogendra Rao Musunuri ${ }^{1}$ (D) and Oh-Seol Kwon ${ }^{2, *}$ (i) \\ 1 Department of Control and Instrumentation Engineering, Changwon National University, \\ Changwon 51140, Korea; musunuri3@gmail.com \\ 2 School of Electrical, Electronics and Control Engineering, Changwon National University, \\ Changwon 51140, Korea \\ * Correspondence: osk1@changwon.ac.kr; Tel.: +82-55-213-3669
}

Citation: Musunuri, Y.R.; Kwon, O.-S. Haze Removal Based on Refined Transmission Map for Aerial Image Matching. Appl. Sci. 2021, 11, 6917. https://doi.org/10.3390/ app11156917

Academic Editor: Antonio Fernández

Received: 14 May 2021

Accepted: 23 July 2021

Published: 27 July 2021

Publisher's Note: MDPI stays neutral with regard to jurisdictional claims in published maps and institutional affiliations.

Copyright: (c) 2021 by the authors. Licensee MDPI, Basel, Switzerland. This article is an open access article distributed under the terms and conditions of the Creative Commons Attribution (CC BY) license (https:// creativecommons.org/licenses/by/ $4.0 /)$.

\begin{abstract}
A novel strategy is proposed to address block artifacts in a conventional dark channel prior (DCP). The DCP was used to estimate the transmission map based on patch-based processing, which also results in image blurring. To enhance a degraded image, the proposed single-image dehazing technique restores a blurred image with a refined DCP based on a hidden Markov random field. Therefore, the proposed algorithm estimates a refined transmission map that can reduce the block artifacts and improve the image clarity without explicit guided filters. Experiments were performed on the remote-sensing images. The results confirm that the proposed algorithm is superior to the conventional approaches to image haze removal. Moreover, the proposed algorithm is suitable for image matching based on local feature extraction.
\end{abstract}

Keywords: dehazing; image-matching; scale invariant feature (SIFT); color transform

\section{Introduction}

Image matching is inevitably challenging when different images of a large scene are being matched. Although local feature points are generally used to match the same location in different images, image matching is difficult in terms of viewpoint, illumination, and temporal changes. There are two types of image-matching method: area-based and feature-based methods [1]. Area-based methods are used to assess the similarity of the pixels between the two images. In feature-based methods, salient features are directly extracted rather than intensity values. In early remote-sensing systems, this work was performed manually. However, this process can now be performed automatically using of local feature point detectors and descriptors [2,3].

Local feature extraction uses the key points, which are generally based on geometric (scale, location, affine) or photometric (illumination, color) approaches. Moreover, most recent algorithms are robust and invariant to image transformations [4]. Therefore, such techniques are widely used in remote-sensing applications [5], such as object detection [6,7] and recognition [8,9], object tracking, scene analysis, classification [10], image matching [11], and retrieval $[12,13]$.

However, in the case of outdoor scenes, image degradation [14] also occurs because of atmospheric conditions, such as haze [15-18], smoke, snow [19], fog [20,21], and rain [22-24]. Haze is particularly problematic for remote-sensing applications [25] when the images are captured from above the earth's atmosphere. Haze is caused by absorption, scattering, and re-emission in the atmosphere. Therefore, in the case of bad weather, images taken with a camera are degraded by haze depending on the distance. As a result, the degraded images lose their contrast and color fidelity.

Conventional methods for removing haze use additional hardware [26-31], multiple images [32], or additional information [33]. Multiple-image-based dehazing methods remove haze through two or more input images. These methods were developed by Tan [34], 
in which haze is removed by maximizing the local contrast based on statistics. However, because this approach is not a physical model, it only provides an improvement. Tarel and Hautiere [35] proposed a fast visibility restoration method to preserve edges and corners based on a median filter; however, this method is not suitable for images with a discontinuous depth. Ancuti et al. [36] recently proposed a simple contrast-based dehazing method without any geometrical information or user interaction. Minseo et al. [37] used principal component analysis to enhance haze images by estimating accurate transmission with a random forest. Dan et al. [38] implemented an efficient quad decomposition algorithm to estimate the atmospheric light to solve the color distortion and background noise problems. This method was applied to synthetic and hazy datasets.

Fattal [39] assumed that the surface shading and transmission functions were locally and statistically uncorrelated to estimate the color of the haze. Moreover, He et al. [40] proposed the DCP method, in which it is assumed that dehazed images have local patches that contain a small number of pixels with low intensity in at least one color channel. However, this method produces block artifacts that are patch based. He et al. [41] also proposed a guided filter-based dehazing method, but this method only focused on edgepreserving filtering. Geet et al. [42] investigated a new color channel method to remove atmospheric scattering, and obtained channel values and atmospheric light. However, this method focuses on enhancing the quality of the dehazed image using a dual transmission map. Chang et al. [43] proposed a method to solve the underestimation of the transmission of brighter areas using a weighted residual map. Yutaro et al. [44] also focused on local patches to address structural detail losses using the normalized pixel-wise DCP. Image dehazing algorithms can be classified as shown by the table in Figure 1.

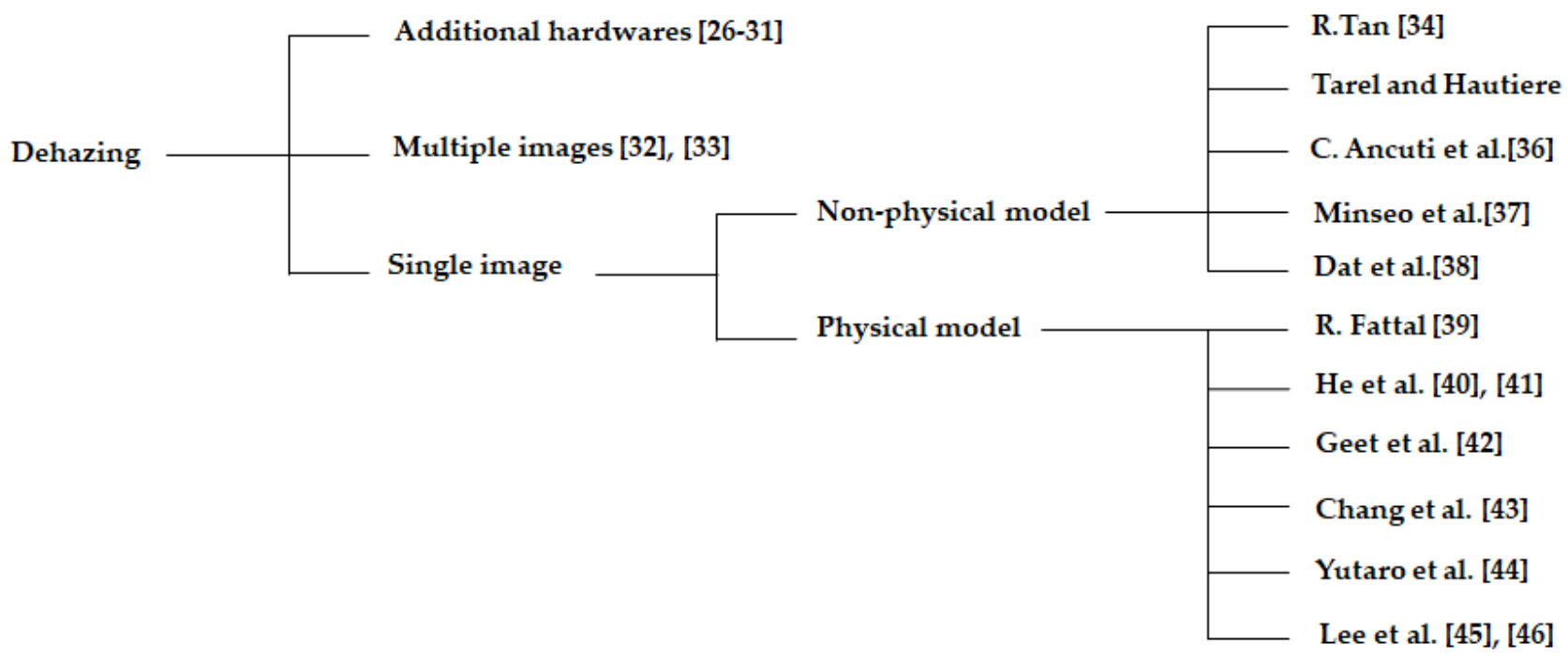

Figure 1. Classification table for image dehazing algorithms [26-46].

In this article, a novel algorithm is introduced that can remove haze from a degraded single image. The proposed single-image-based algorithm restores a hazy image by refining a transmission map using an approach based on a hidden Markov model $[45,46]$. The proposed strategy enhances the image contrast by reducing the block artifacts from the patch-based image dehazing method. As a second contribution, the proposed de-hazing method is suitable for image matching based on local feature-embedded colors using the C-SIFT operator. Finally, image matching was evaluated using aerial-haze images, the $\mathrm{NH}-\mathrm{Haze}$ dataset, and a manually annotated dataset. The remainder of this article is organized as follows. In Section 2, a refined transmission map using the proposed method and its implementation are described. In Section 3, the experimental results are presented and discussed. Section 4 concludes the work. Additionally, the assessment of the dehazed images was evaluated and compared using the PIQE [47]. 


\section{Physical-Based Image Dehazing and Matching}

Haze is a challenging weather phenomenon for remote-sensing aerial images because, in dehazing, scene transmission depends on unknown depth information. The physical model removes haze based on direct transmission maps. To address block artifacts, a novel method is proposed to estimate a refined transmission map without explicit guided filters. The method consists of two parts. The first is calculation of the DCP, and estimation of the atmospheric light and DCP transmission map. The transmission map of DCP is then refined using the hidden Markov random field and expectation maximization (HMRF-EM) algorithm, which is explained in Sections 2.1 and 2.2. Second, image-matching is performed using the scale-invariant feature transform (SIFT) algorithm to find the valid key-point matches, which is explained in Section 2.3.

\subsection{Dark Channel Prior}

In general, the method of modeling the haze in one image is expressed by Equation (1), which defines the haze image, original image, atmospheric light, and transmission.

$$
I(x)=J(x) t(x)+A(1-t(x))
$$

where $I(x)$ is an image containing haze, and $J(x)$ is the brightness value of the original image without haze. $A$ is the atmospheric light and $t(x)$ is the transmission, which shows the degree to which the unseen light reaches the camera.

The DCP [40] is based on the observation in dehazed outdoor images of a few pixels whose intensity values in at least one color channel are very low in non-sky patches, namely, red (R), green, $(\mathrm{G})$, or blue (B); that is, the patch is composed of very low values with minimum intensity and the dark channel is defined in Equation (2).

$$
J^{d a r k}(x)=\min _{c \in\{r, g, b\}}\left(\min _{y \in \Omega(x)}\left(J^{c}(y)\right)\right)
$$

where $J^{C}$ is the color channel of $J$, and $\Omega(x)$ is a local patch centered at $x$. Based on observation, with the exception of the sky region, the intensity of $J^{\text {dark }}$ is low and tends to be zero if $J$ is a haze-free outdoor image. Thus, $J^{\text {dark }}$ is the dark channel of $J$, and the above statistical observation is the DCP. To remove haze in the image, the top $0.1 \%$ of the brightest pixels were obtained for the estimated atmospheric light in the dark channel, and the chosen pixels were haze-opaque. In the next step, the coarse estimated transmission is as follows:

$$
\widetilde{t}(x)=1-\omega \min _{c \in\{r, g, b\}}\left(\min _{y \in \Omega(x)}\left(\frac{J^{c}(y)}{\widetilde{A}^{c}}\right)\right)
$$

where $\widetilde{A}^{c}$ is the color channel of estimated atmospheric light, and $\omega$ is chosen as $(0<\omega<1)$ [40] to prevent the full elimination of haze because it causes the haze to result in an unnatural image with the loss of depth perception. We fixed the value of $\omega$ to 0.95 for all subsequent experimental results in this study. The estimated transmission map $\widetilde{t}$ is refined by applying a soft-matting method. Finally, the estimated scene radiance is expressed as:

$$
\widetilde{J}(x)=\frac{J(x)-\widetilde{A}}{\max \left(\widetilde{t}(x), t_{0}\right)}+\widetilde{A}
$$

where $t_{0}$ is the lower limit of the refined medium transmission and $\widetilde{A}$ is the estimated atmospheric light. When applying the steps of the DCP method, the haze removal results can be obtained. Algorithm 1 provides the procedure for each step, as follows, to estimate the scene radiance $\widetilde{J}$. 


\subsection{Correction of Transmission Map Using Hidden Markov Random Field}

The application of an HMRF-EM algorithm $[45,46,48]$ to estimate the transmission map is proposed. The HMRF-EM algorithm improves the transmission map and enhances the dehazing performance based on a double stochastic process that uses the maximum likelihood to remove and smooth the areas of block artifacts. The input image is represented by $\mathrm{y}=\left(y_{1}, \ldots, y_{N}\right)$ when the intensity is $y_{i}$. The labels $\mathrm{x}=\left(x_{1}, \ldots, x_{N}\right)$ are estimated using the HMRF method. Here, $x_{i} \in L$ and $L$ is the set of possible block artifact labels.

$$
\mathbf{x}^{*}=\underset{\mathbf{x}}{\operatorname{argmax}}[P(\mathbf{y} \mid \mathbf{x}, \theta) P(\mathbf{x})]
$$

where $P(\mathbf{x})$ is the prior probability that represents the Gibbs distribution and the probability of joint maximum-likelihood is expressed as follows:

$$
P(\mathbf{y} \mid \mathbf{x}, \theta)=\prod_{i} P\left(y_{i} \mid \mathbf{x}, \theta\right)=\prod_{i} P\left(y_{i} \mid x_{i}, \theta_{x_{i}}\right)
$$

Here, $P\left(y_{i} \mid x_{i}, \theta_{i}\right)$ is the Gaussian distribution of the variable $\theta_{x_{i}}=\left(\mu_{x_{i}}, \sigma_{x_{i}}\right)$, and $\theta=\left\{\theta_{l} \mid l \in L\right\}$ is the variable set from the EM method. After determining the variable set, the Gaussian distribution function $G\left(z, \theta_{l}\right)$ can be represented using $\theta=\left(\theta_{l}, \sigma_{l}\right)$ as follows:

$$
G\left(z ; \theta_{l}\right)=\frac{1}{\sqrt{2 \pi \sigma_{l}^{2}}}\left(-\frac{\left(z-\mu_{l}\right)^{2}}{2 \sigma_{l}^{2}}\right)
$$

Next, two assumptions are required that are used to approximate the proposed HMRFEM algorithm. The prior probability is defined as:

$$
P(\mathbf{x})=\frac{1}{Z} \exp (-U(\mathbf{x}))
$$

where $U(\mathbf{x})$ denotes the prior energy function. The second assumption is as follows:

$$
\begin{gathered}
P(\mathbf{y} \mid \mathbf{x}, \theta)=\prod_{i} P\left(y_{i} \mid x_{i}, \theta_{x_{i}}\right) \\
=\prod_{i} G\left(y_{i} ; \theta_{x_{i}}\right) \\
=\frac{1}{Z^{\prime}} \exp (-U(\mathbf{y} \mid \mathbf{x}))
\end{gathered}
$$

From these two assumptions, the variable $\theta^{(0)}$ is initialized. The probability distribution $P^{(t)}\left(y_{i} \mid x_{i}, \theta_{x_{i}}\right)$ is also estimated. The labels are then estimated using $\theta^{(0)}$. During this process, the estimation of the maximum a posteriori (MAP) probability is utilized, as shown below.

$$
\begin{aligned}
& \mathbf{x}^{(t)} \underset{x \in \chi}{\operatorname{argmax}}\left[P\left(\mathbf{y} \mid \mathbf{x}, \theta^{(t)}\right) P(\mathbf{x})\right] \\
& =\underset{x \in \chi}{\operatorname{argmax}}\left[U\left(\mathbf{y} \mid \mathbf{x}, \theta^{(t)}\right)+U(\mathbf{x})\right]
\end{aligned}
$$

Next, the posterior distributions of all $l \in L$ and $y_{i}$ are calculated as follows:

$$
P^{(t)}\left(l \mid y_{i}\right)=\frac{G\left(y_{i} ; \theta_{l}\right) P\left(l \mid x_{N_{i}}^{(t)}\right)}{P^{(t)}\left(y_{i}\right)}
$$

where $x_{N}^{(t)}$ is the neighbor of $x_{i}^{(t)}$.

$$
P^{(t)}\left(y_{i}\right)=\sum_{l \in L} G\left(y_{i} ; \theta_{l}\right) P\left(l \mid x_{N_{i}}^{(t)}\right)
$$


and

$$
P\left(l \mid x_{N_{i}}^{(t)}\right)=\frac{1}{Z} \exp \left(-\sum_{j \in N_{i}} V_{c}\left(l, x_{j}^{(t)}\right)\right)
$$

Next, the variables are updated using the $P^{(t)}\left(l \mid y_{i}\right)$ as follows:

$$
\begin{gathered}
\mu_{l}^{(t+1)}=\frac{\sum_{i} P^{(t)}\left(l \mid y_{i}\right) y_{i}}{\sum_{i} P^{(t)}\left(l \mid y_{i}\right)} \\
{\left[\sigma_{l}^{(t+1)}\right]^{2}=\frac{\sum_{i} P^{(t)}\left(l \mid y_{i}\right)\left(y_{i}-\mu_{l}^{(t+1)}\right)^{2}}{\sum_{i} P^{(t)}\left(l \mid y_{i}\right)}}
\end{gathered}
$$

Equations (5)-(15) are related to the HMRF-EM method. Equations (16)-(20) are related to MAP estimation and edge preservation. Equation (16) is defined to solve $\mathbf{x}^{*}$ and minimize the posterior energy [48].

$$
\mathbf{x}^{*}=\underset{x \in \chi}{\operatorname{argmax}}[U(\mathbf{y} \mid \mathbf{x}, \theta)+U(x)]
$$

The likelihood energy is defined in Equation (17) as follows:

$$
U(\mathbf{y} \mid \mathbf{x}, \theta)=\sum_{i} U\left(y_{i} \mid x_{i}, \theta\right)=\sum_{i}\left[\frac{\left(y_{i}-\mu_{x_{i}}\right)^{2}}{2 \sigma_{x_{i}}{ }^{2}}+\ln \sigma_{x_{i}}\right]
$$

The prior energy is defined using $U(x)$, as shown in Equation (18).

$$
U(x)=\sum_{c \in C} V_{c}(x)
$$

The clique potential is defined as in (19) for the neighboring pixels.

$$
V_{c}\left(x_{i}, x_{j}\right)=\frac{1}{2}\left(1-I_{x_{i}, x_{j}}\right)
$$

From the above equations, we obtain Equation (20) to smooth the edges of the block artifacts, as follows:

$$
x_{i}^{(k+1)}=\underset{l \in L}{\operatorname{argmax}}\left[U\left(y_{i} \mid l\right)+\sum_{j \in N_{i}, z_{j}=0} V_{c}\left(l \mid x_{j}^{(k)}\right)\right]
$$

where $\mathbf{z}$ is the assumption of the binary edge map (if $z_{i}=1$, the edge is not deteriorated; otherwise, the edge is deteriorated) and $V_{c}$ is the clique potential. Algorithm 1 explains each step to obtain a refined transmission map using the HMRF-EM method.

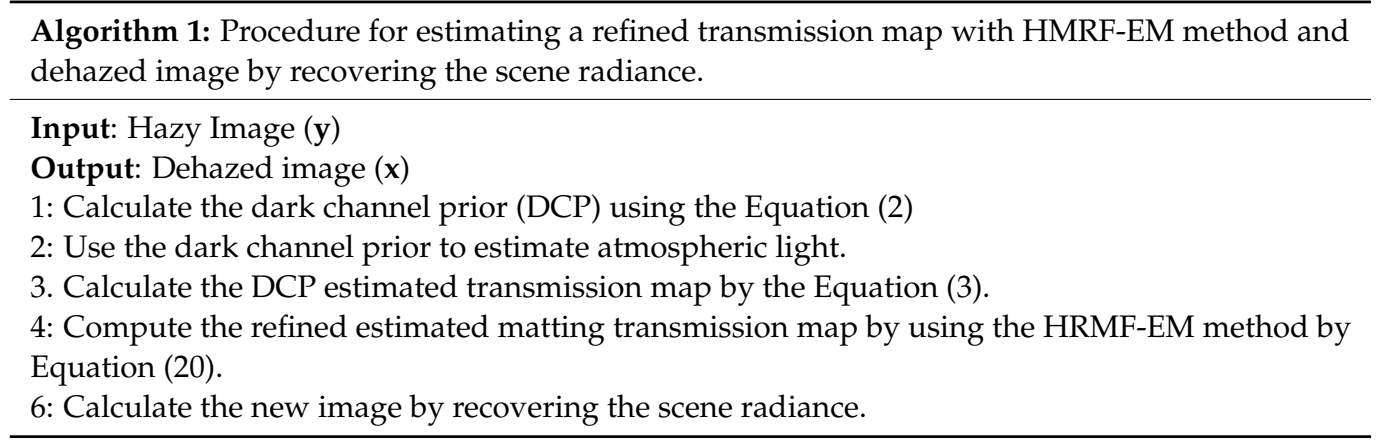




\subsection{Scale Invariant Feature Transform with Embedded Color}

The SIFT [49] operator is broadly used in image matching due to its robustness to the rotation of the image, scaling, and variations in luminance. This algorithm consists of the following five steps: scale-space extrema detection, key-point localization, orientation assignment, key-point descriptor, and key-point matching. Moreover, SIFT with embedded color (C-SIFT) [50] was used in this study. The C-SIFT algorithm is robust because the local features include color information for image dehazing. In general, the camera response, as shown in (17), is given by [51]:

$$
\rho_{i}^{x y}=\int_{w}\left\{L_{P}(\lambda)+E(\lambda) R(\lambda)\right\} S_{i}(\lambda) d \lambda, \quad(i=R, G, B)
$$

where $\rho_{i}$ is the response of the camera, $\lambda$ is the wave length of the color, $L_{p}(\lambda)$ is the path radiance, $E(\lambda)$ is the illumination, $R(\lambda)$ is the surface reflectance, and $S(\lambda)$ is the sensitivity of the camera. In addition, the $x y$-coordinates are allocated in the array to every point.

$$
\rho_{i}^{x y}=\int_{w} L_{P}(\lambda) E(\lambda) R(\lambda) S_{i}(\lambda) d \lambda+\zeta_{i}^{x y}, \quad(i=R, G, B)
$$

here,

$$
\zeta_{i}^{x y}=\int_{w} L_{P}(\lambda) S_{i}(\lambda) d \lambda,(i=R, G, B)
$$

Although haze cannot be entirely removed by dehazing processing, it is assumed that $\zeta_{i}^{x y}$ is not a dominant component. Here, if it is assumed that the camera sensitivities are delta functions, Equation (21) can be revised for any wavelength $k$. Note that realistic camera sensitivities are not delta functions, which is an issue that is discussed later.

$$
\begin{gathered}
\rho_{k}^{x y}=\int_{w} E(\lambda) R(\lambda) \delta\left(\lambda-\lambda_{k}\right) d \lambda \\
=E\left(\lambda_{k}\right) R\left(\lambda_{k}\right)
\end{gathered}
$$

The color response at the Planckian locus of an illuminant for any pixel is defined as follows:

$$
\rho_{k}=c_{1} \lambda_{k}{ }^{-5} \exp \left(\frac{-c_{2}}{T \lambda_{k}}\right) R\left(\lambda_{k}\right)
$$

where

$$
E(\lambda, T)=c_{1} \lambda^{-5}\left[\exp \left(\frac{-c_{2}}{T \lambda}\right)-1\right]^{-1}
$$

Here, $c_{1}=3.74183 \times 10^{-16} \mathrm{Wm}^{2}$ and $c_{2}=1.4388 \times 10^{-2} \mathrm{mK}$. The natural logarithm is used to separate the two components, namely, the surface and illuminant, using Equation (24) as follows:

$$
\ln \rho_{k}=\ln \left[c_{1} \lambda_{k}{ }^{-5} R\left(\lambda_{k}\right)\right]-\frac{c_{2}}{T \lambda_{k}}
$$

Following the determination of the color responses for each RGB channel, the ratio for each channel is calculated using the same process as follows:

$$
\begin{aligned}
& \ln \frac{R}{G}=\ln \rho_{R}-\ln \rho_{G}=R_{R}-R_{G}+\frac{1}{T}\left(E_{R}-E_{G}\right) \\
& \ln \frac{B}{G}=\ln \rho_{B}-\ln \rho_{G}=R_{B}-R_{G}+\frac{1}{T}\left(E_{B}-E_{G}\right)
\end{aligned}
$$

where $R_{k}=\ln \left[c_{1} \lambda_{k}{ }^{-5} R\left(\lambda_{k}\right)\right]$ and $E_{k}=-c_{2} / \lambda_{k}$. The simple transform is used to identify the two significant clues using Equation (26).

$$
\ln \frac{R}{G}-\frac{E_{R}-E_{G}}{E_{B}-E_{G}} \ln \frac{B}{G}=R_{R}-R_{G}+\frac{E_{R}-E_{G}}{E_{B}-E_{G}}\left(R_{B}-R_{G}\right)
$$


Equation (27) shows that the relationship between the coordinates of the log-chromaticity and the altered level in the illuminant is linear, whereas $R_{k}$ is not dependent on the illuminant color. In addition, the relationship between the log-chromaticity and change in the surface reflectance is also linear, as shown in Equation (29).

$$
\left(\ln \frac{R}{G}-\frac{R_{R}-R_{G}}{R_{B}-R_{G}} \ln \frac{B}{G}\right) T=E_{R}-E_{G}-\frac{R_{R}-R_{G}}{R_{B}-R_{G}}\left(E_{B}-E_{G}\right)
$$

When using a real camera, because it is impossible to implement the abovementioned sensitivities using a delta function, the proposed method uses the channel filter function of Worldview-2 to consider the characteristics of aerial images, rather than the mathematical approach presented in Equations (22)-(29). Next, the transformation of a real camera response into a new response is explained. A linear regression model [52] is used. Finally, 1485 Munsell patches for which the color-invariant values are normalized at the minimum and maximum values are used as the input values of the C-SIFT as shown in Algorithm 2.

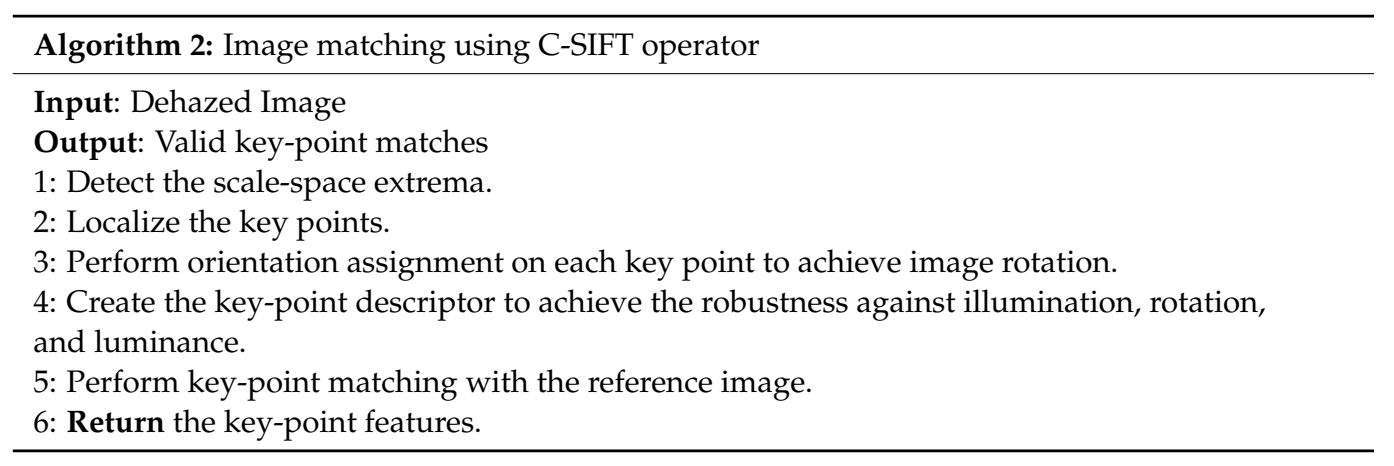

\section{Results and Discussion}

The experiments were performed on four datasets to evaluate the performance of the proposed algorithm, namely, synthetic haze images, natural aerial images from Google, NH-HAZE [53,54], and manually annotated Datasets. NH-HAZE is a synthetic nonhomogeneous haze dataset consisting of 55 outdoor images. The manually annotated dataset was created in the lab and consists of 18 images for testing the homogeneous aerial images. We present the results in Figures 2-9. The experimental results show that the proposed method performs well on all the datasets. Generally, haze-free images use a disparity map to generate synthetic haze images. The results of the different methods are shown in Figure 2. The results for the method of He et al. show some block artifacts in certain regions. When observed using a uniform transmission map, the details of the transmission map cause image blur. However, the proposed algorithm reduced the block artifacts in the transmission map and enhanced the haze removal performance. Figure 3 shows the test images and the results obtained using the proposed method with aerial images. To evaluate the proposed method in Figures 3-5, natural aerial images were obtained from Google. The image sizes were $3264 \times 2448,4318 \times 3083,950 \times 691$, and $3008 \times 2000$. The performance of the proposed algorithm was also compared with those of several state-of-the-art dehazing methods when using aerial images, as shown in Figure 4. The proposed algorithm was used to process and analyze several large aerial images with a resolution of $3000 \times 3000$ pixels. The Figure 4 are the input hazy images are compared with the proposed method and conventional methods such as Tarel et al. [35], and $\mathrm{He}$ et al. [40,41]. The proposed un-optimized (MATLAB implementation) code could process each aerial image in approximately half an hour using a desktop computer (Intel 5, 3.1-GHz CPU, 8-GB RAM). The proposed method produced visually pleasing results that were superior to those produced by conventional methods. The results obtained using the proposed algorithm were generally sharper and clearer than those obtained using the method of He et al. Although the image features could be easily detected when the image contrast was high, the method of Tarel and Hautiere [35] performed poorly. 

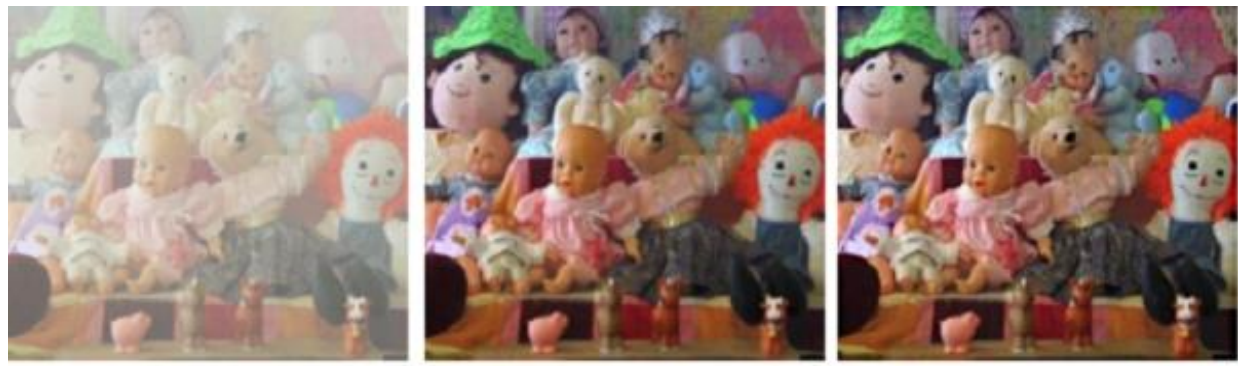

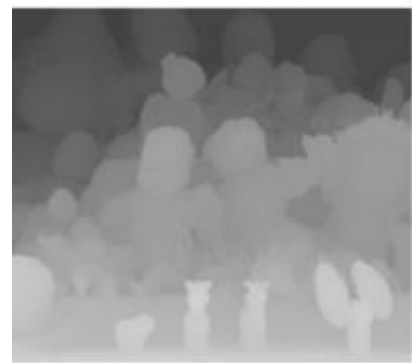

(a)

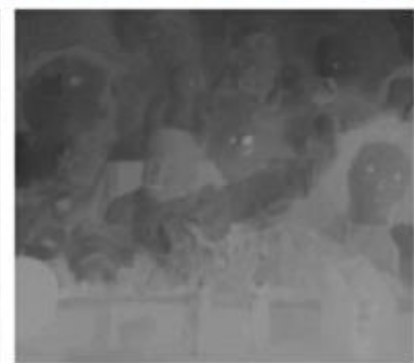

(b)

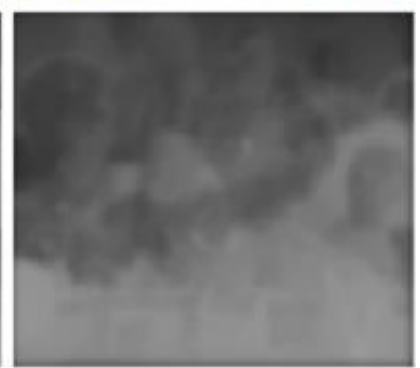

(c)

Figure 2. Images for each algorithm and their transmission maps: (a) hazy image, (b) He et al. [40] method, and (c) proposed method.

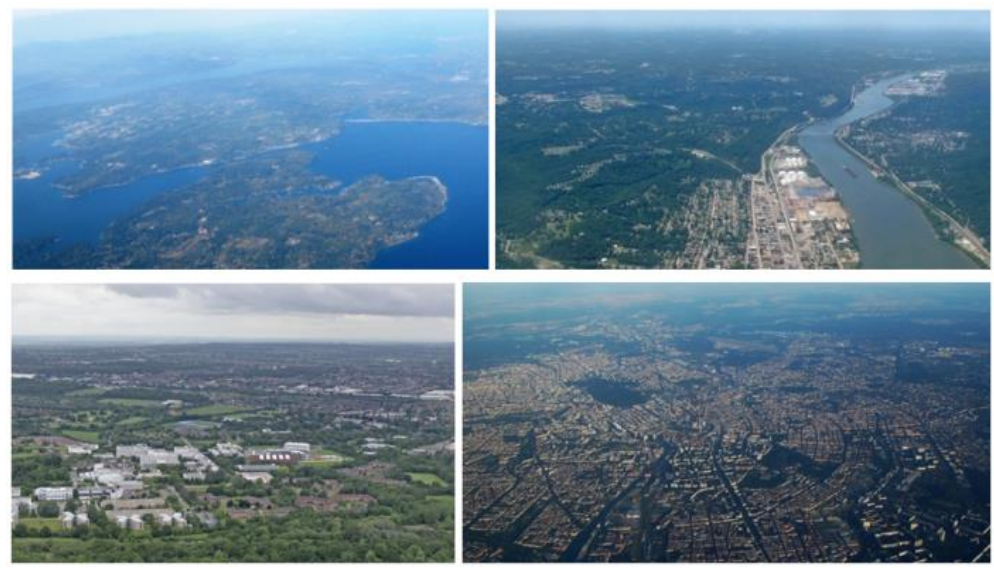

(a)
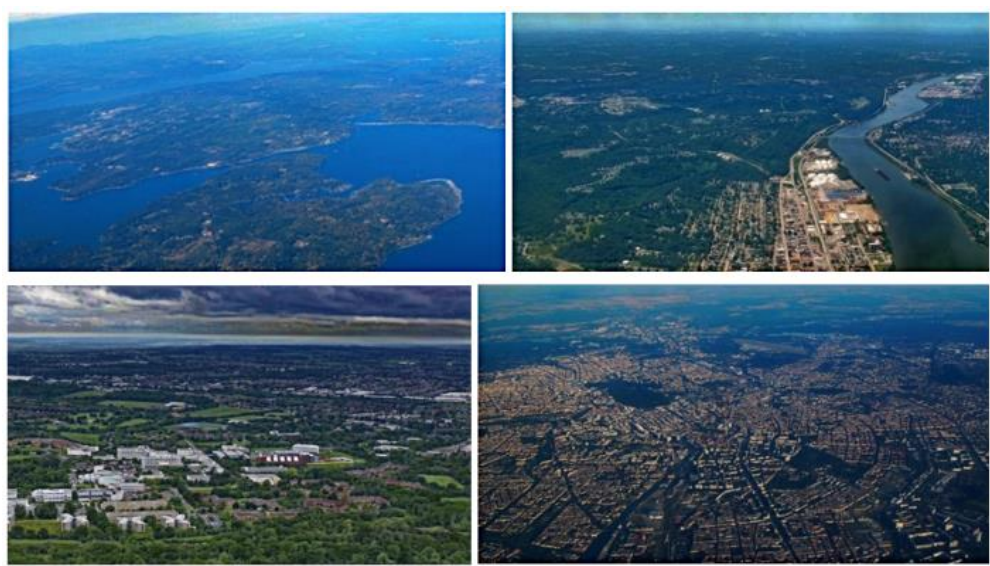

(b)

Figure 3. Test aerial images and dehazing result with large sizes: (a) original hazy images and (b) proposed method. 


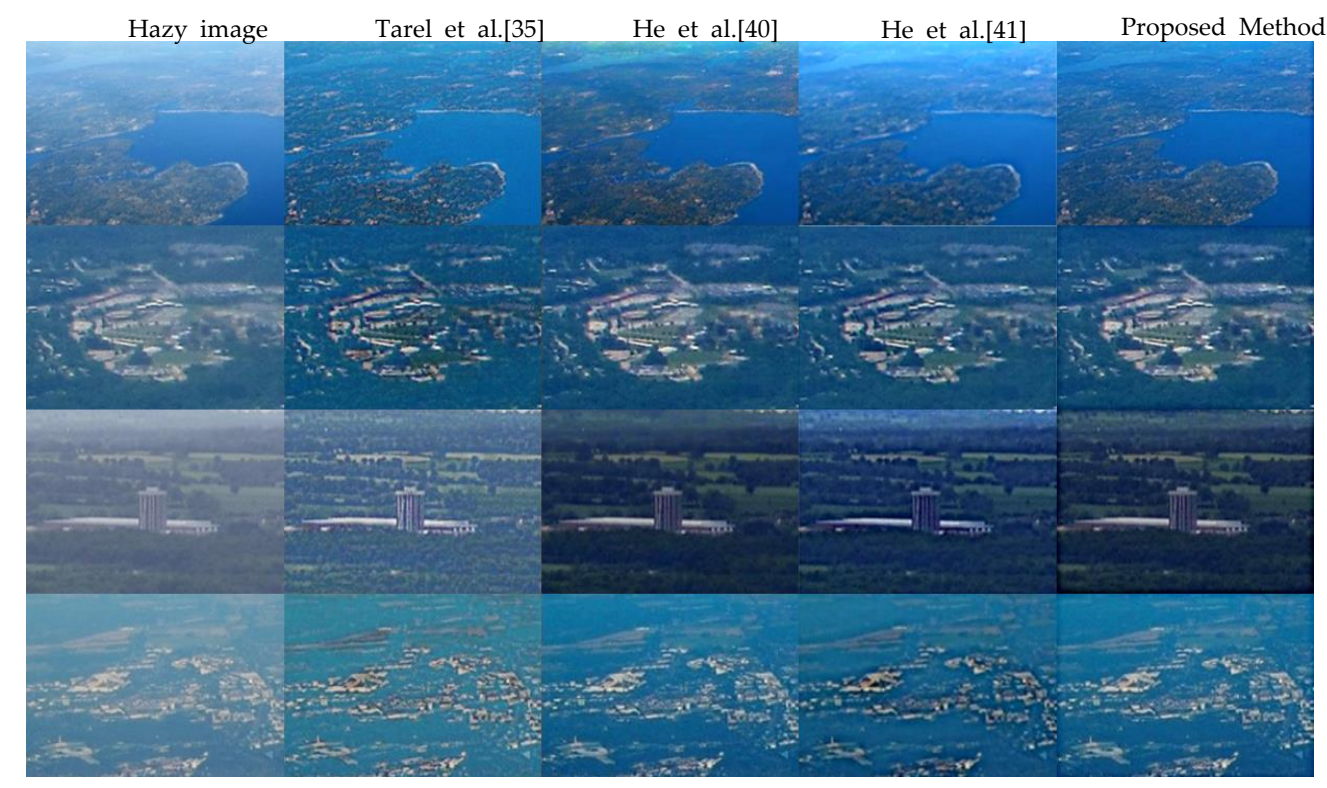

Figure 4. Results of several algorithms for aerial images.

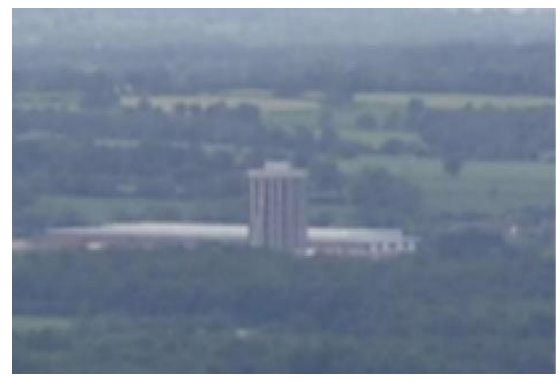

(a)

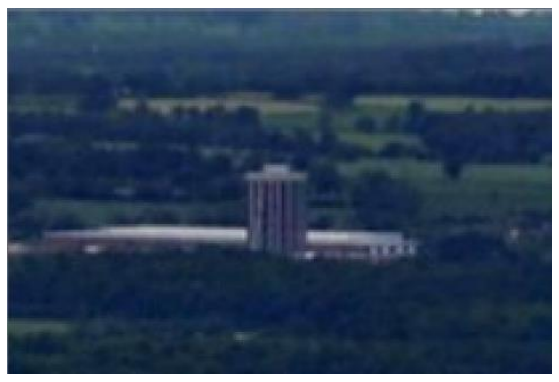

(c)

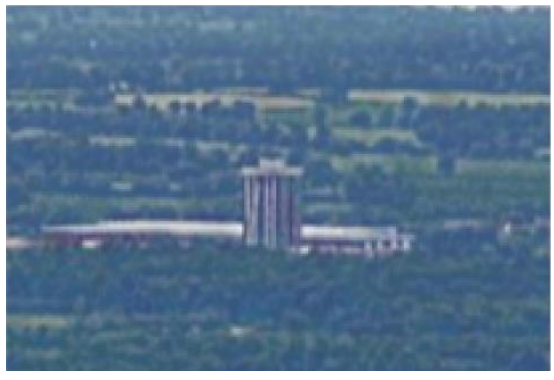

(b)

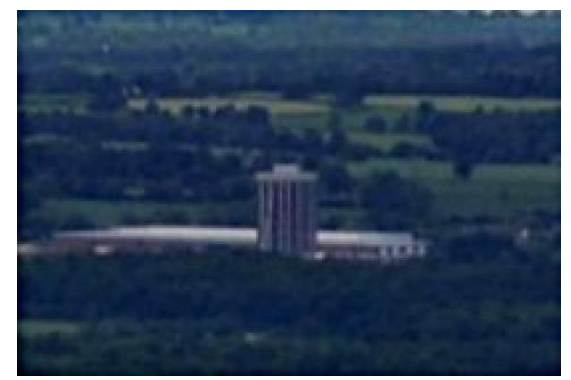

(d)

Figure 5. Detailed images of Figure 4: (a) original hazy image, (b) Tarel and Hautiere [35] method, (c) He et al. [40] method, and (d) proposed method.

Figure 5 shows the detailed images based on magnifying the images in Figure 4 . Figure $5 c$, d shows a block problem caused by block processing, whereas Figure $5 b$ does not show the block problem. Block artifacts can occur in the case of mixed clouds and haze (e.g., very bright regions) when using block-based processing. However, the proposed algorithm reduces the number of block artifacts, as shown in Figure 5d. The reduction of block artifacts is not clear in the aerial images of the proposed method. Therefore, we compared their transmission maps, as shown in Figure 6. We can clearly see how the block artifacts have been smoothed compared to the DCP. For example, Figure 6a is a DCP transmission map containing block artifacts, and Figure $6 \mathrm{~b}$ is a refined transmission map using the proposed method with reduced block artifacts. However, some of these may be affected by uncertainties; therefore, they require fuzzy preprocessing [55-57]. Figures 7 
and 8 show the results for non-homogeneous and homogeneous images. Also, the Figures 7 and 8 are the input hazy images are compared with the proposed method and conventional methods such as Tarel et al. [35], and He et al. [40,41].

(a)

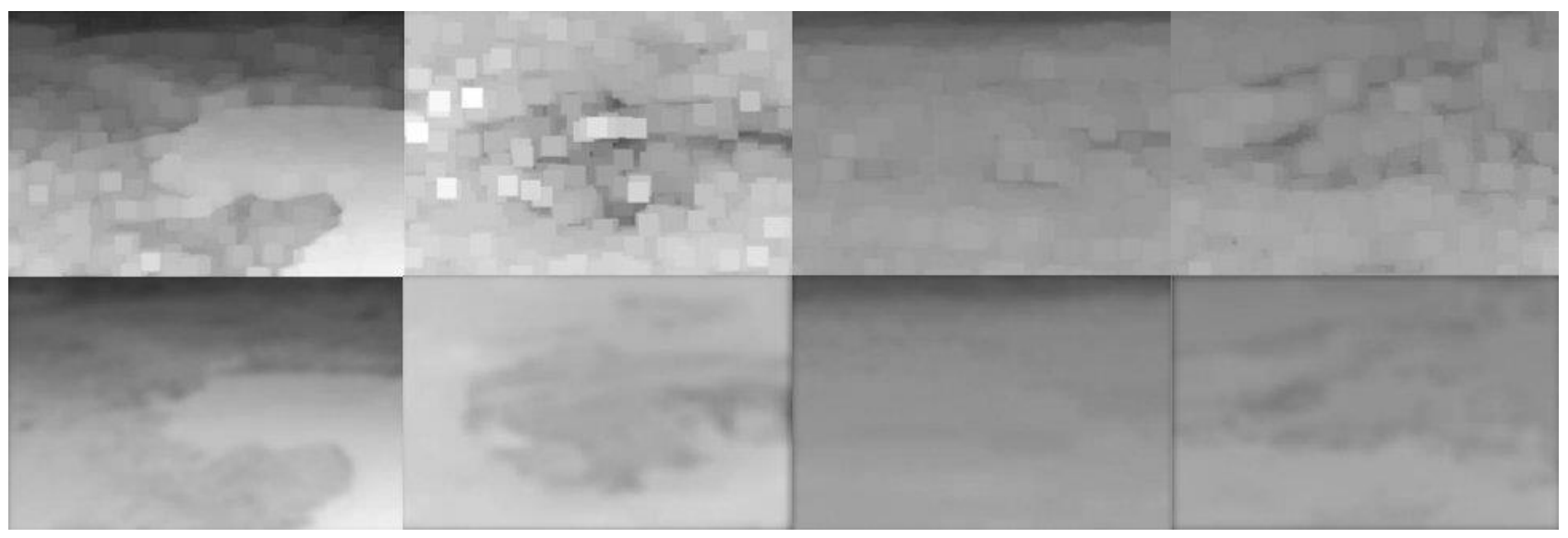

Figure 6. Transmission map of Figure 4: (a) DCP transmission map, (b) refined transmission map of the proposed method.

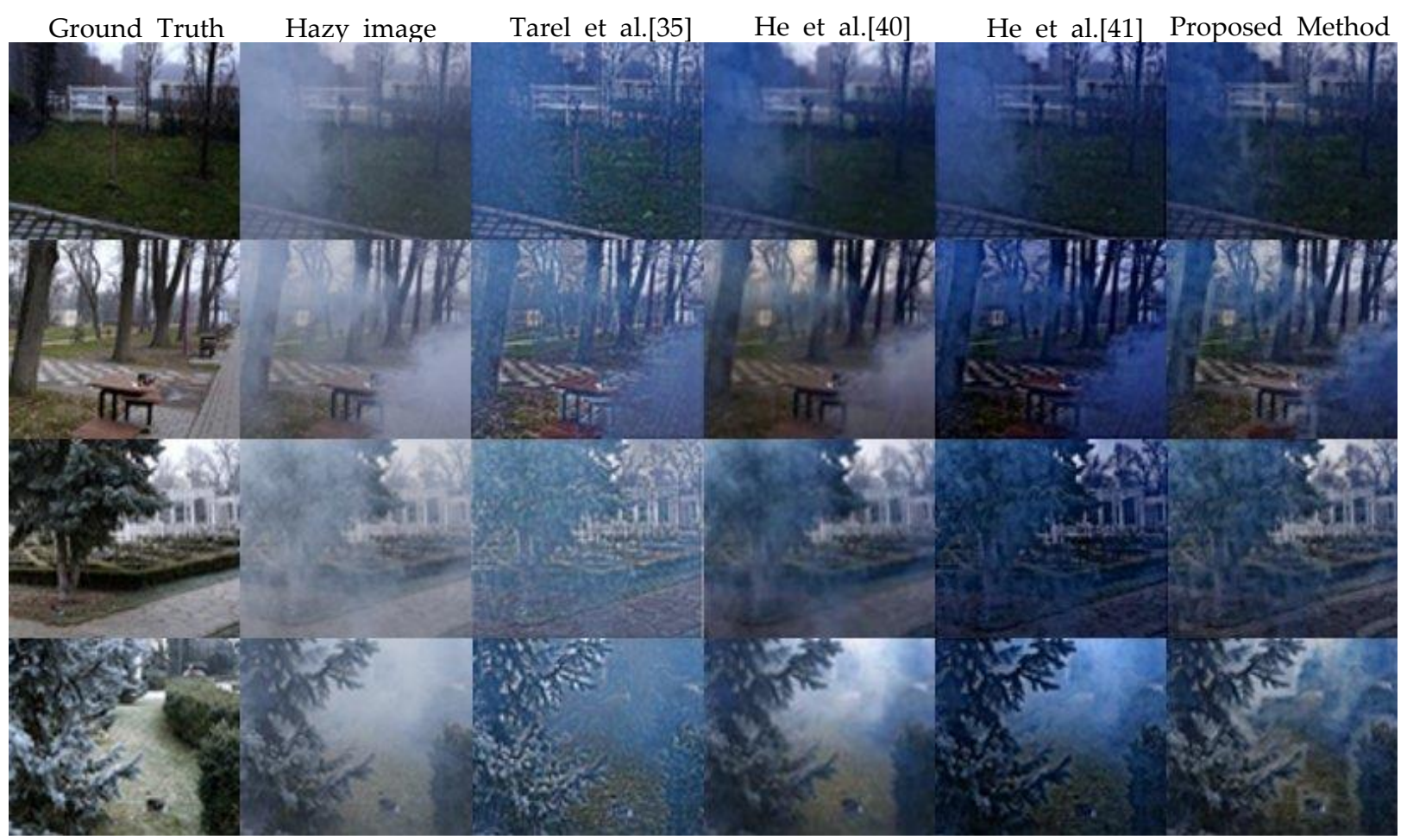

Figure 7. Comparative test results of different dehazing methods using NH-HAZE dataset with Tarel and Hautiere [35], He et al. [40], He et al. [41], and the proposed method.

The proposed approach and the conventional methods, as listed in Table 1, were compared with the help of the PIQE [47] metric to evaluate the quality of the de-hazed image. The PIQE metric is used for human perceptual quality, which is inversely correlated with perceptual quality. Thus, a low score indicates good perceptual quality, and a high score indicates poor perceptual quality. The bold text indicates the best performance and the dark-green text indicates the next-best performance. For instance, the low value for the first image in Figure 4 is that of the proposed method, with 15.5900, and the highest value is that of $\mathrm{He}$ et al. [41], with 34.5783. The same procedure was followed for the other tested images. Of all values of the metric, the proposed method demonstrates the 
best performance. The bold text indicates the best performance and the dark-green text indicates the next-best performance. Hence, the results suggest that the proposed method yields better results on tested datasets and aerial images.

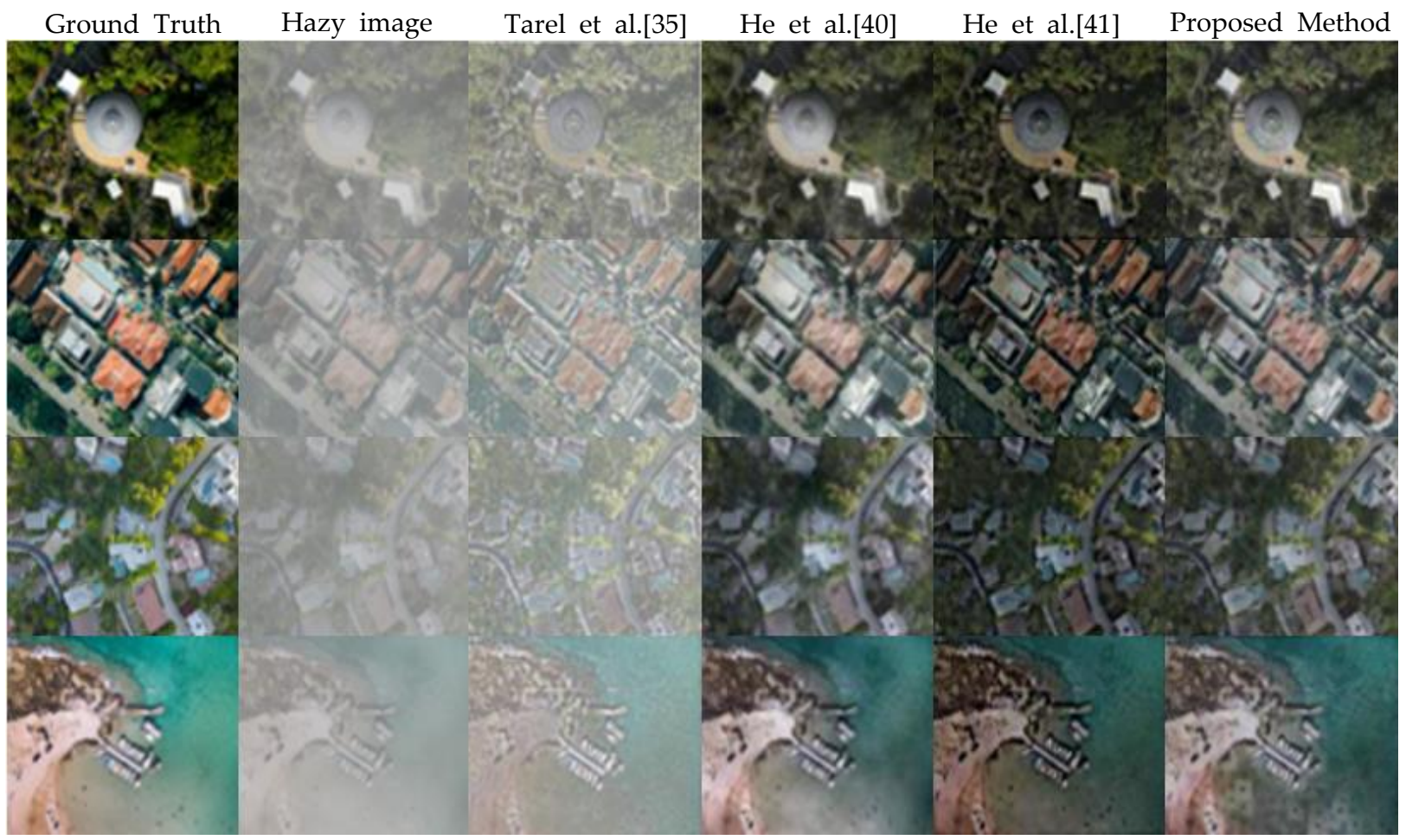

Figure 8. Comparative test results of different dehazing methods using manually annotated dataset with Tarel and Hautiere [35], He et al. [40], He et al. [41], and the proposed method.

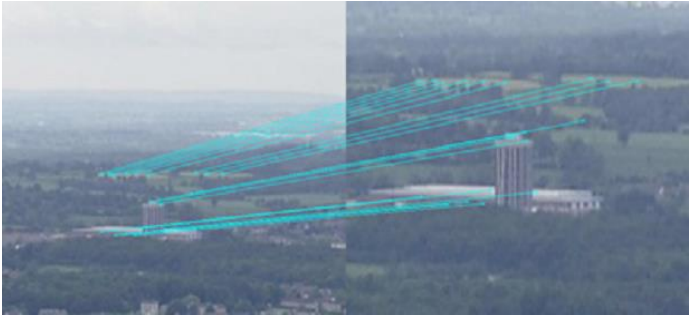

(a)

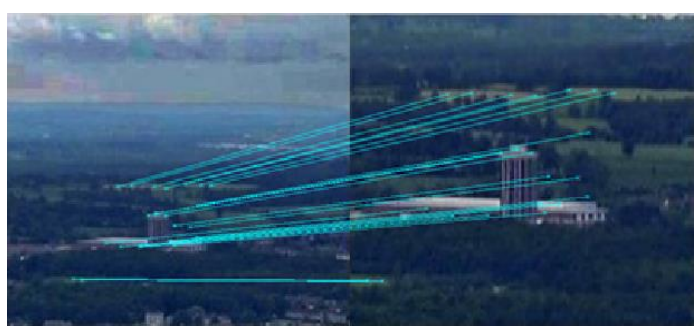

(c)

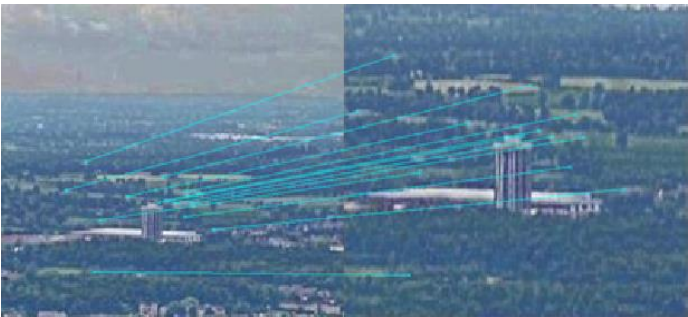

(b)

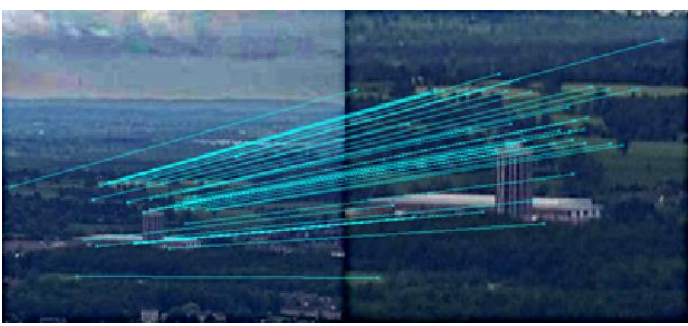

(d)

Figure 9. Matching based on C-SIFT: (a) original haze images (32 good matches), (b) Tarel and Hautiere [35] method (31 good matches) , (c) He et al. [40] method (74 good matches), and (d) proposed method (85 good matches). 
Table 1. Quantitative comparison of different methods based on PIQE evaluation.

\begin{tabular}{cccccc}
\hline S. No & Tested Image & Tarel et al. [35] & He et al. [40] & He et al. [41] & $\begin{array}{c}\text { Proposed } \\
\text { Method }\end{array}$ \\
\hline 1 & Figure 4 & 20.8522 & 23.7638 & 34.5783 & $\mathbf{1 5 . 5 9 0 0}$ \\
2 & Figure 4 & 26.1828 & 27.0469 & 36.8676 & $\mathbf{2 1 . 5 3 1 3}$ \\
3 & Figure 4 & 41.0633 & 50.6249 & 53.1791 & $\mathbf{3 3 . 1 9 8 7}$ \\
4 & Figure 4 & 31.3141 & 27.8593 & 39.0135 & $\mathbf{2 5 . 7 7 9 6}$ \\
5 & Figure 7 & 40.0932 & 61.6822 & 34.7334 & $\mathbf{2 9 . 7 8 1 1}$ \\
6 & Figure 7 & 40.8646 & 65.8164 & 39.7221 & $\mathbf{3 5 . 5 1 0 1}$ \\
7 & Figure 7 & $\mathbf{3 3 . 0 5 2 5}$ & 70.4645 & 34.9615 & 34.7679 \\
8 & Figure 7 & 36.9200 & 47.7217 & 36.3100 & $\mathbf{2 6 . 1 6 2 7}$ \\
9 & Figure 8 & $\mathbf{2 5 . 8 4 1 4}$ & 72.7626 & 28.4863 & 28.7927 \\
10 & Figure 8 & 26.0668 & 60.1055 & 26.7333 & $\mathbf{2 2 . 1 9 3 6}$ \\
11 & Figure 8 & $\mathbf{2 6 . 5 6 9 1}$ & 76.9486 & 31.8643 & 30.3427 \\
12 & Figure 8 & 32.6156 & 60.5336 & 34.9176 & $\mathbf{3 2 . 0 3 5 9}$ \\
\hline
\end{tabular}

Finally, the effect of enhancing the image contrast by eliminating the haze in an image is emphasized by the image-matching results shown in Figure 9. For the C-SIFT pointmatching results, an experiment using a significant number of test images was conducted to support the point-matching related claims. When the C-SIFT operator was applied to the original hazy images, only 32 valid matches were produced. When the C-SIFT operator was applied to images enhanced using the method of Tarel and Hautiere [35], 31 valid matches were obtained. When the C-SIFT operator was applied to images enhanced using the method of He et al. [40], 74 valid matches resulted. Finally, when the proposed method based on C-SIFT was used, 85 valid matches were generated. Additionally, we show the valid key point matching techniques compared to different methods in Table 2. Of these methods, the proposed technique demonstrates more valid matches due to the reduction in block artifacts.

Table 2. Quantitative comparison of feature-points matching obtained from different methods.

\begin{tabular}{cccccc}
\hline S. No & Tested Image & Tarel et al. [35] & He et al. [40] & He et al. [41] & $\begin{array}{c}\text { Proposed } \\
\text { Method }\end{array}$ \\
\hline 1 & Figure 4 & 24 & $\mathbf{1 0 2}$ & 62 & 92 \\
2 & Figure 4 & 55 & 217 & 184 & $\mathbf{2 5 4}$ \\
3 & Figure 4 & 31 & 74 & 46 & $\mathbf{8 5}$ \\
4 & Figure 4 & 152 & 351 & 203 & $\mathbf{3 5 2}$ \\
5 & Figure 7 & 17 & 13 & 19 & $\mathbf{3 0}$ \\
6 & Figure 7 & 18 & 56 & 25 & $\mathbf{7 1}$ \\
7 & Figure 7 & 08 & 35 & 15 & $\mathbf{5 5}$ \\
8 & Figure 7 & 29 & 67 & 72 & $\mathbf{1 0 8}$ \\
9 & Figure 8 & 52 & 132 & 66 & $\mathbf{1 2 3}$ \\
10 & Figure 8 & 114 & 17 & 471 & $\mathbf{4 0 5}$ \\
11 & Figure 8 & 10 & 56 & 82 & $\mathbf{7 4}$ \\
12 & Figure 8 & 58 & & & $\mathbf{1 7 7}$ \\
\hline
\end{tabular}

\section{Conclusions}

A dehazing method for image contrast enhancement was proposed. The method modifies the transmission map based on the DCP using the HMRF-EM algorithm. The method can enhance the image contrast by reducing the block artifacts caused by a patchbased image dehazing algorithm. The performance of the proposed algorithm was found to be superior to that of conventional methods for synthetic, outdoor, and aerial images. The algorithm is suitable for image-matching based on local feature points and outperforms conventional methods. The algorithm has certain limitations. For example, it can only be applied to RGB-based images, excluding satellite images without a blue band. The performance of the algorithm is also degraded when haze affects near-infrared channels. 
The performance of the delta functions, one of the basic assumptions of the proposed algorithm, can also be adversely affected when realistic camera sensitivities are used.

Author Contributions: Conceptualization, Y.R.M. and O.-S.K.; methodology, Y.R.M. and O.-S.K.; investigation, Y.R.M. and O.-S.K.; writing-original draft preparation, Y.R.M. and O.-S.K.; writingreview and editing, Y.R.M. and O.-S.K.; supervision, O.-S.K.; project administration, O.-S.K. All authors have read and agreed to the published version of the manuscript.

Funding: This research was supported by Basic Science Research Program through the National Research Foundation of Korea (NRF) funded by the Ministry of Science, ICT \& Future Planning (2019R1F11058489).

Institutional Review Board Statement: Not applicable.

Informed Consent Statement: Not applicable.

Data Availability Statement: The publicly archived NH-HAZE dataset is available at https:/ / data. vision.ee.ethz.ch/cvl/ntire20/nh-haze (accessed on 26 July 2021) and manually annotated dataset is generated during the study which is available at https:/ / unsplash.com/s/photos/aerial (accessed on 26 July 2021).

Conflicts of Interest: The authors declare no conflict of interest.

\section{References}

1. Leng, C.; Zhang, H.; Li, B.; Cai, G.; Pei, Z.; He, L. Local feature descriptor for image matching: A survey. IEEE Access 2019, 7, 6424-6434. [CrossRef]

2. Xi, W.; Shi, Z.; Li, D. Comparisons of feature extraction algorithm based on unmanned aerial vehicle image. Open Phys. 2017, 15, 472-478. [CrossRef]

3. Zhang, C.-M.; Gong, Z.-H.; Huang, Y. Performance evaluation and improvement of several feature point detectors. J. Geomat. Sci. Technol. 2008, 25, 231-234.

4. Tuytelaars, T.; Mikolajczyk, K. Local invariant feature detectors: Survey. Found. Trends Comput. Graph. Vis. 2008, 3, 177-280.

5. Zhou, H.; Dai, A.; Tian, T.; Tian, Y.; Yu, Z.; Wu, Y.; Zhang, Y. Feature matching for remote sensing image registration via manifold regularization. IEEE J. Sel. Top. Appl. Earth Obs. Remote. Sens. 2020, 13, 4564-4574. [CrossRef]

6. Halavataya, K. Local feature descriptor indexing for image matching and object detection in real-time applications. Pattern Recognit. Image Anal. 2020, 30, 16-21. [CrossRef]

7. Farooq, J. Object detection and identification using SURF and BoW model. In Proceedings of the 2016 International Conference on Computing, Electronic and Electrical Engineering (ICE Cube), Quetta, Pakistan, 11-12 April 2016.

8. Rani, R.; Grewal, S.K.; Panwar, K. Object recognition: Performance evaluation using SIFT and SURF. Int. J. Comput. Appl. 2013, 75, 39-47. [CrossRef]

9. Chen, Y.; Xu, W.; Piao, Y. Target matching recognition for satellite image based on the improved FREAK algorithm. Math. Probl. Eng. 2016, 2016, 1-9. [CrossRef]

10. Li, K.; Wang, F.; Zhang, L. A new algorithm for image recognition and classification based on improved bag of features algorithm. Optik 2016, 127, 4736-4740. [CrossRef]

11. Ma, D.; Lai, H.-c. Remote Sensing Image Matching Based Improved ORB in NSCT Domain. J. Indian Soc. Remote Sens. 2019, 47, 801-807. [CrossRef]

12. Jahangiri, M.; Petrou, M. An attention model for extracting components that merit identification. In Proceedings of the 2009 16th IEEE International Conference on Image Processing (ICIP), Cairo, Egypt, 7-10 November 2009.

13. Harel, J.; Koch, C.; Perona, P. Graph-based visual saliency. In Proceedings of the Twentieth Annual Conference on Neural Information Processing Systems, Vancouver, BC, Canada, 4-7 December 2006.

14. Tao, S.; Feng, H.; Xu, Z.; Li, Q. Image degradation and recovery based on multiple scattering in remote sensing and bad weather condition. Opt. Express 2012, 20, 16584-16595. [CrossRef]

15. Long, J.; Shi, Z.; Tang, W.; Zhang, C. Single remote sensing image dehazing. IEEE Geosci. Remote. Sens. Lett. 2013, 11, 59-63. [CrossRef]

16. Moro, G.D.; Halounova, L. Haze removal for high-resolution satellite data: A case study. Int. J. Remote Sens. 2007, 28, 2187-2205. [CrossRef]

17. Pan, X.; Xie, F.; Jiang, Z.; Yin, J. Haze removal for a single remote sensing image based on deformed haze imaging model. IEEE Signal Process. Lett. 2015, 22, 1806-1810. [CrossRef]

18. Q Zhu, Q.; Mai, J.; Shao, L. A Fast single Image Haze Removal Algorithm Using Color Attenuation Prior. IEEE Trans. Image Process. 2015, 24, 3522-3533.

19. Kawarabuki, H.; Onoguchi, K. Snowfall detection in a foggy scene. In Proceedings of the 2014 22nd International Conference on Pattern Recognition, Stockholm, Sweden, 24-28 August 2014. 
20. Wang, L.; Zhu, R. Image Defogging algorithm of single color image based on wavelet transform and histogram equalization. Appl. Math. Sci. 2013, 7, 3913-3921.

21. Xu, Z.; Liu, X.; Ji, N. Fog removal from color using contrast limited adaptive histogram equalization. In Proceedings of the 2009 2nd International Congress on Image and Signal Processing, Tianjin, China, 17-19 October 2009.

22. Bissonnette, L.R. Imaging through fog and rain. Opt. Eng. 1992, 5, 1045-1052. [CrossRef]

23. Sun, S.-H.; Fan, S.-P.; Wang, Y.-C.F. Exploiting image structural similarity for single image rain removal. In Proceedings of the 2014 IEEE International Conference on Image Processing (ICIP), Paris, France, 27-30 October 2014.

24. You, S.; Tan, R.T.; Kawakami, R.; Ikeuchi, K. Adherent raindrop detection and removal in video. In Proceedings of the 2013 IEEE Conference on Computer Vision and Pattern Recognition, Portland, OR, USA, 23-28 June 2013.

25. Wierzbicki, D.; Kedzierski, M.; Sekrecka, A. A Method for dehazing images obtained from low altitudes during high-pressure fronts. Remote. Sens. 2020, 12, 25. [CrossRef]

26. Namer, E.; Shwartz, S.; Schechner, Y.Y. Skyless polarimetric calibration and visibility enhancement. Opt. Express 2009, 17, 472-493. [CrossRef]

27. Kopf, J.; Neubert, B.; Chen, B.; Cohen, M.; Cohen-Or, D.; Deussen, O.; Uyttendaele, M.; Lischinski, D. Deep photo: Model-based photo-graph enhancement and viewing. ACM Trans. Graphpics 2008, 27, 1-10. [CrossRef]

28. Shen, L.; Zhao, Y.; Peng, Q.; Chan, J.C.-W.; Kong, S.G. An iterative image dehazing method with polarization. IEEE Trans. Multimed. 2019, 21, 1093-1107. [CrossRef]

29. Liang, Z.; Ding, X.; Mi, Z.; Wang, Y.; Fu, X. Effective polarization-based image dehazing with regularization constant. IEEE Geosci. Remote Sens. Lett. 2020, 18, 1-5. [CrossRef]

30. Reda, M.; Zhao, Y.; Chan, J.C.-W. Polarization guided autoregressive model for depth recovery. IEEE Photonics J. 2017, 9, 1-16. [CrossRef]

31. Wang, X.; Ouyang, J.; Wei, Y.; Liu, F.; Zhang, G. Real-time vision through haze based on polarization imaging. Appl. Sci. 2019, 9, 142. [CrossRef]

32. Narasimhan, S.G.; Nayar, S.K. Contrast restoration of weather degraded image. IEEE Trans. Pattern Anal. Mach. Intell. 2003, 25, 713-724. [CrossRef]

33. Makarau, A.; Richter, R.; Muller, R.; Reinartz, P. Haze detection and removal in remotely sensed multispectral imagery. IEEE Trans. Geosci. Remote Sens. 2014, 52, 5895-5905. [CrossRef]

34. Tan, R.T. Visibility in bad weather from a single image. In Proceedings of the 2008 IEEE Conference on Computer Vision and Pattern Recognition, Anchorage, AK, USA, 23-28 June 2008.

35. Tarel, J.P.; Haultiere, N. Fast visibility restoration from a single color or gray level image. In Proceedings of the 2009 IEEE 12 th International Conference on Computer Vision, Kyoto, Japan, 29 September-2 October 2009.

36. Ancuti, C.; Ancuti, C.O. Effective contrast-based dehazing for robust image matching. IEEE Geosci. Remote. Sens. Lett. 2014, 11, 1871-1875. [CrossRef]

37. Kim, M.; Yu, S.; Park, S.; Lee, S.; Paik, J. Image dehazing and enhancement using principal component analysis and modified haze features. Appl. Sci. 2018, 8, 1321. [CrossRef]

38. Ngo, D.; Lee, G.-D.; Kang, B. Improved color attenuation prior for single-image haze removal. Appl. Sci. 2019, 9, 4011. [CrossRef]

39. Fattal, R. Single image dehazing. ACM Trans. Graph. 2008, 27, 1-10. [CrossRef]

40. He, K.; Sun, J.; Tang, X. Single image haze removal using dark channel prior. IEEE Trans. Pattern Anal. Mach. Intell. 2011, 33, 2341-2353.

41. He, K.; Sun, J.; Tang, X. Guided image filtering. IEEE Trans. Pattern Anal. Mach. Intell. 2013, 35, 1397-1409. [CrossRef] [PubMed]

42. Sahu, G.; Seal, A.; Krejcar, O.; Yazidi, A. Single image dehazing using a new color channel. J. Vis. Commun. Image Represent. 2021, 74, 1-16. [CrossRef]

43. Su, C.; Wang, W.; Zhang, X.; Jin, L. Dehazing with offset correction and a weighted residual map. Electronics 2020, 9, 1419. [CrossRef]

44. Iwamoto, Y.; Hashimoto, N.; Chen, Y.-W. Real-time haze removal using normalized pixel-wise dark-channel prior and robust atmospheric-light estimation. Appl. Sci. 2020, 10, 1165. [CrossRef]

45. Lee, M.-H.; Kwon, O.-S. Image dehazing using transmission map based on hidden markov random field model. J. Inst. Electron. Inf. Eng. 2014, 51, 145-151.

46. Lee, M.-H.; Kwon, O.-S. Image haze removal based on transmission map using hidden markov random field model. IEICE Trans. Fundam. Electron. Commun. Comput. Sci. 2014, E97-A, 1820-1822. [CrossRef]

47. Musunuri, Y.R.; Kwon, O.-S. Deep residual dense network for single image super-resolution. Electronics 2021, 10, 555. [CrossRef]

48. Wang, Q. Hmrf-em Image: Implementation of the Hidden Markov Random Field Model and its Expectation-Maximization Version 2.0. Available online: http:/ / arxiv.org/abs/1207.3510 (accessed on 10 June 2020).

49. Lowe, D. Distinctive image features from scale-invariant key points. Int. J. Comput. Vis. 2004, 60, 91-110. [CrossRef]

50. Kwon, O.-S.; Ha, Y.-H. Panoramic Video using Scale-Invariant Feature Transform with Embedded Color-Invariant Values. IEEE Trans. Consum. Electron. 2010, 56, 792-798. [CrossRef]

51. Liang, S.; Fang, H.; Chen, M. Atmospheric correction of Landsat ETM+ land surface imagery-Part I: Methods. IEEE Trans. Geosci. Remote Sens. 2001, 39, 2490-2498. [CrossRef]

52. Lee, H. Introduction to Color Imaging Science; Cambridge University Press: Cambridge, UK, 2005. 
53. Ancuti, C.O.; Ancuti, C.; Timofte, R. NH-HAZE: An image dehazing benchmark with non-homogeneous hazy and haze-free images. In Proceedings of the IEEE CVPR NTIRE Workshop, Seattle, WA, USA, 14-19 June 2020.

54. Ancuti, C.O.; Ancuti, C.; Vasluianu, F.A.; Timofte, R. NTIRE 2020 challenge on non-homogeneous dehazing. In Proceedings of the IEEE CVPR NTIRE Workshop, Seattle, WA, USA, 14-19 June 2020.

55. Toğaçar, M.; Ergen, B.; Cömert, Z. COVID-19 detection using deep learning models to exploit Social Mimic Optimization and structured chest X-ray images using fuzzy color and stacking approaches. Comput. Biol. Med. 2020, 121, 1-12. [CrossRef] [PubMed]

56. Narmatha, C.; Sarah Mustafa, E.; Mohammed Tuka, A.R.; Manimurugan, S.; Mustafa, M. A hybrid fuzzy brain-storm optimization algorithm for the classification of brain tumor MRI images. J. Ambient. Intell. Humaniz. Comput. 2020, 5, 1-9. [CrossRef]

57. Versaci, M.; Morabito, F.C.; Angiulli, G. Adaptive Image Contrast Enhancement by Computing Distances into a 4-Dimensional Fuzzy Unit Hypercube. IEEE Access 2017, 5, 26922-26931. [CrossRef] 